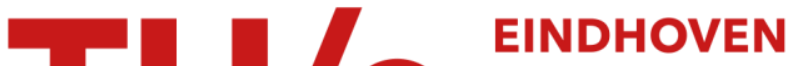 UNIVERSITY OF TECHNOLOGY
}

\section{An Accurate Characterization Method for Integrated Polarization Converters}

\section{Citation for published version (APA):}

Reniers, S., Williams, K. A., van der Tol, J. J. G. M., \& Jiao, Y. (2021). An Accurate Characterization Method for Integrated Polarization Converters. IEEE Journal of Quantum Electronics, 57(1), [9311824].

https://doi.org/10.1109/JQE.2020.3047119

DOI:

10.1109/JQE.2020.3047119

Document status and date:

Published: 01/02/2021

\section{Document Version:}

Accepted manuscript including changes made at the peer-review stage

\section{Please check the document version of this publication:}

- A submitted manuscript is the version of the article upon submission and before peer-review. There can be important differences between the submitted version and the official published version of record. People interested in the research are advised to contact the author for the final version of the publication, or visit the $\mathrm{DOI}$ to the publisher's website.

- The final author version and the galley proof are versions of the publication after peer review.

- The final published version features the final layout of the paper including the volume, issue and page numbers.

Link to publication

\section{General rights}

Copyright and moral rights for the publications made accessible in the public portal are retained by the authors and/or other copyright owners and it is a condition of accessing publications that users recognise and abide by the legal requirements associated with these rights.

- Users may download and print one copy of any publication from the public portal for the purpose of private study or research.

- You may not further distribute the material or use it for any profit-making activity or commercial gain

- You may freely distribute the URL identifying the publication in the public portal.

If the publication is distributed under the terms of Article 25fa of the Dutch Copyright Act, indicated by the "Taverne" license above, please follow below link for the End User Agreement:

www.tue.nl/taverne

Take down policy

If you believe that this document breaches copyright please contact us at:

openaccess@tue.nl

providing details and we will investigate your claim. 


\title{
An Accurate Characterization Method for Integrated Polarization Converters
}

\author{
Sander F.G. Reniers, Kevin A. Williams, Jos J.G.M. van der Tol, Yuqing Jiao
}

\begin{abstract}
We present a characterization method for polarization converters which improves the accuracy of traditional characterization methods significantly. An experimental demonstration of the method is presented on the InP-membrane-onsilicon (IMOS) platform. The design and fabrication of the polarization converter is discussed, as well as the simulated polarization conversion efficiency. A device of only 4 microns is shown to achieve $97.5 \% \pm 0.5 \%$ polarization conversion, corresponding to an extinction ratio of $-16 \pm 0.9 \mathrm{~dB}$. The traditional characterization method is compared to the new 4-port method, and the accuracy is improved from up to $20 \%$ to $0.5 \%$.
\end{abstract}

Index Terms-Photonic integrated circuits, nanophotonics, photonic crystals, polarization, polarization converters.

\section{INTRODUCTION}

$\mathbf{F}$ IBER-OPTIC communication was developed in the $1970 \mathrm{~s}$, and has revolotionized the telecommunications industry. The high bandwidth capabilities of optical fibers have made them an alternative to traditional copper cables that could not be overlooked. New technologies, e.g. wavelength division multiplexing, polarization diversity schemes and advanced digital coherent technologies increased the popularity of optical communication even more. Traditionally, optical transceivers were used for long distance communication or for bandwidths exceeding the capabilities of electronics. More recently, optical communications are also used for short distance communication, e.g. rack-to-rack interconnects in data centers. Low power consumption and integratability with electronics is of paramount importance nowadays. Integrated polarization converters enable polarization diversity schemes such as DPWDM and more complex schemes, e.g. Stokes vector modulation. For these purposes, it is of paramount importance to have precise control over the conversion efficiency. To be able to control the conversion efficiency, one needs to have the tools to characterize the converters as accurately as possible, which are the characterization circuits. Traditionally, on platforms that offer edge-coupling, an arbitrary state of polarization can be coupled in and out of the chip. With proper analysis, the conversion efficiency can be extrapolated from such measurements, when making use of polarizers and waveplates. However, on nanophotonic platforms, edgecoupler is not a conventional method for coupling light, simply due to the mismatch of the mode size of the fundamental

Manuscript received August 14, 2020. This work was supported in part by the Dutch Zwaartekracht Grant. The use of the cleanroom facilities at the Nanolab@TU/e is acknowledged.

The authors are with the Institute of Photonic Integration (IPI), Eindhoven. University of Technology, P.O.Box 513, 5600 MB Eindhoven, The Netherlands, (e-mail: s.f.g.reniers@tue.nl). modes in the photonic wires and the optical fiber. Therefore, surface grating couplers, which are operating for either the TE or the TM polarization with high extinction to the orthogonal polarization, are typically used for this purpose. Generally, transmission measurements are dependent on the insertion loss of these surface grating couplers, which can vary based on location on the chip, e.g. due to slight thickness variations in the BCB thickness. Furthermore, one needs to compensate the measurement for those insertion losses by using calibration structures, which introduces uncertainties in the characterization.

We introduce a method for characterization of polarization converters which is independent on the insertion loss of the grating couplers and other components in the characterization circuit, and hence boosts the accuracy of the characterization significantly. This is an adjustment to the method that is used to characterize photonic crystal reflectors [1]. The key difference is that for the characterization of polarization converters, there is only one unknown, the conversion efficiency, instead of two unknowns for the reflectors, the transmission and reflection of a reflector. Therefore, the accuracy for the characterization of polarization converters is even higher.

The InP-membrane-on-silicon (IMOS) platform [2] offers many advantages for demonstration of this characterization method. Firstly, the unique triangular shaped structure is extremely compact [3]. Secondly, the platform offers full active-passive integration, making this method a useful tool for further development. Lastly, the methodology that is reported here can be transferred to any photonic integration platform. The IMOS platform consists of a $300 \mathrm{~nm}$ thick membrane of InP, which corresponds to the core thickness in the waveguides, bonded by benzocyclobutene (BCB) to a carrier wafer (typically silicon or CMOS). An example of a cross section of an IMOS waveguide is illustrated in Fig. 1. The core thickness and high index contrast enable tight light confinement and device miniaturization. A description of the fabrication process of IMOS devices, and specifically the polarization converters, is given in Section II-A.

\section{POLARIZATION CONVERTER}

In this section, the polarization converter that is used for demonstration of the characterization method is presented. Firstly, the design and the fabrication process are discussed. Secondly, the performance of the device in a simulation is shown. 


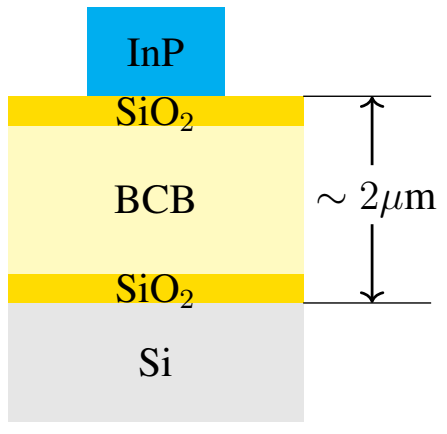

Fig. 1. Cross section of a typical waveguide on the IMOS platform. Dimensions of the InP waveguide: $400 \mathrm{~nm}$ wide, $300 \mathrm{~nm}$ thick.

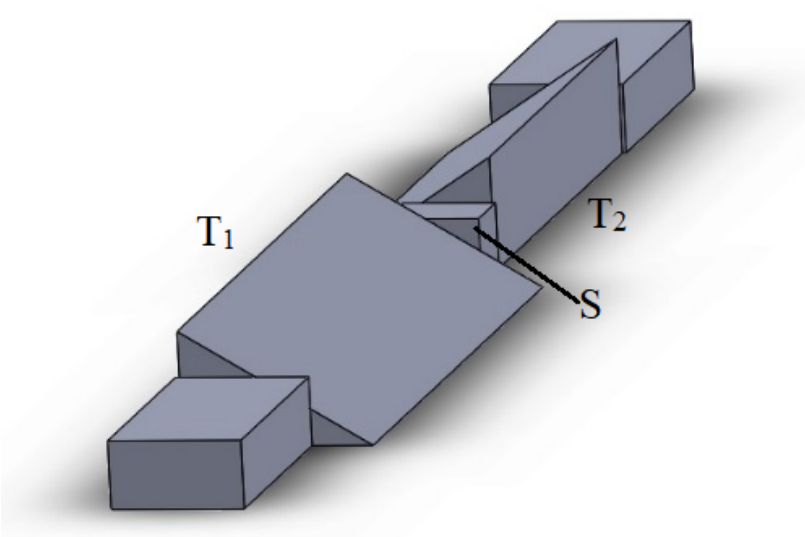

Fig. 2. Design of the polarization converter.

\section{A. Design and fabrication}

The design of the polarization converter is shown in Fig. 2. It consists of two triangular sections, which are connected by a short rectangular waveguide. The working principle is based on mode hybridization [3]. To achieve optimal mode coupling between the rectangular and triangular sections, the height of the triangular sections is larger than the standard core thickness of $300 \mathrm{~nm}$. To achieve the slope of the triangular sections, $\mathrm{HCl}: \mathrm{H}_{3} \mathrm{PO}_{4}(1: 4)$ is used as a wet etchant, which results in a slope of 35 degrees. An additional etch-stoplayer is introduced, such that only in the areas where the triangular sections are present the extra thick membrane exists, whereas on the rest of the wafer the membrane is only $300 \mathrm{~nm}$ thick. A separate lithographic step is required to define these areas. To create the slope in only one direction, the vertical sidewall of the polarization converter is etched first, in the same lithographic step as the waveguide definition. The area around the triangular section is then opened in the consecutive lithographic step, and the silicon nitride hardmask is etched directionally, ensuring that the vertical sidewall is still covered by silicon nitride. Since the $\mathrm{HCl}: \mathrm{H}_{3} \mathrm{PO}_{4}(1: 4)$ wet etch stops on the $\{\overline{1} \overline{1} 2\}$ crystal plane of InP [4], the triangular section is formed. A cartoon view of the key steps in the fabrication process is shown in Fig. 4. Note that the flexibility of the IMOS platform provides the option of processing the InP on

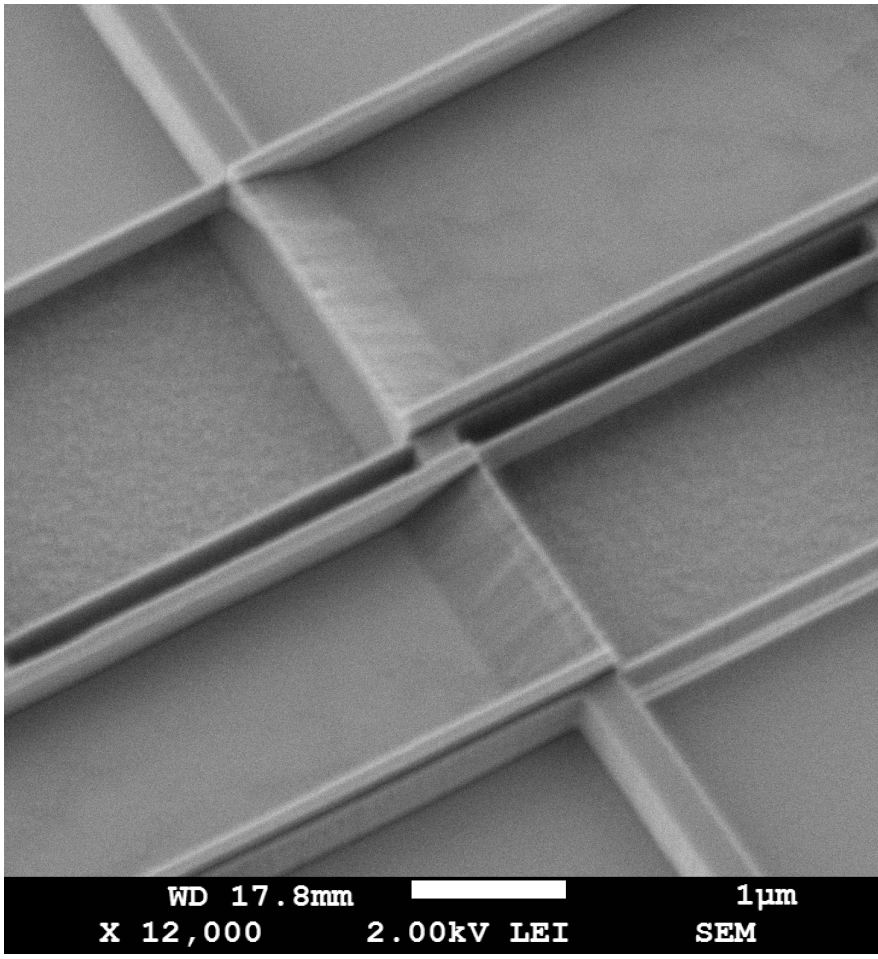

Fig. 3. Scanning electron microscope image of a fabricated polarization converter before bonding.

both sides, before and after the bonding process. In this process flow, the choice is made to perform all the processing before the bonding process, since this enables integration of the component in the twin-guide active-passive integration scheme of IMOS. A scanning electron microscope (SEM) image of a fabricated device, taken before wafer bonding, is shown in Fig. 3.

\section{B. Simulation}

To determine the dimensions of the triangular sections, both eigenmode expansion (EME) and finite-difference timedomain (FDTD) simulations are performed. Firstly, the optimal height for the triangular sections is determined, by calculating the best overlap of the hybrid modes in these sections with the modes in the standard IMOS waveguide (400 $\mathrm{nm}$ wide by $300 \mathrm{~nm}$ high). This height was found to be $460 \mathrm{~nm}$, and thus the thickness for the extra layers shown in Fig. 4 are $20 \mathrm{~nm}$ for the etch-stop-layer, and $140 \mathrm{~nm}$ for the extra indium phosphide layer.

Secondly, the hybrid modes in the triangular sections need to be analyzed. The two hybrid modes that exist in this $460 \mathrm{~nm}$ high triangular section are shown in Fig. 5. The left mode has an effective index of 2.269 , the right mode has an effective index of 1.901. From the TE fraction of one of the modes, the tilt angle of the mode is calculated by $\theta=\cos ^{-1}(\sqrt{0.78}) \approx 28^{\circ}$. This result indicates a single section device is not sufficient to achieve full polarization conversion, since the maximum achievable rotation for a single section device is $2 \cdot 28=56^{\circ}$. Therefore, a double section device is chosen, and hence any state of polarization can be achieved. Since we are interested 


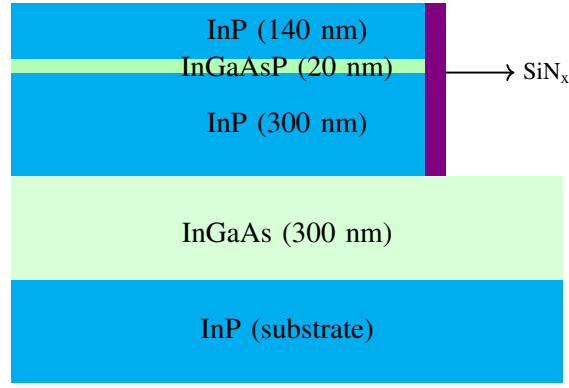

(a)

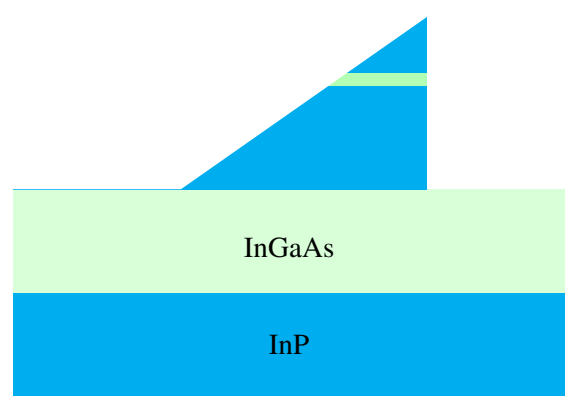

(b)

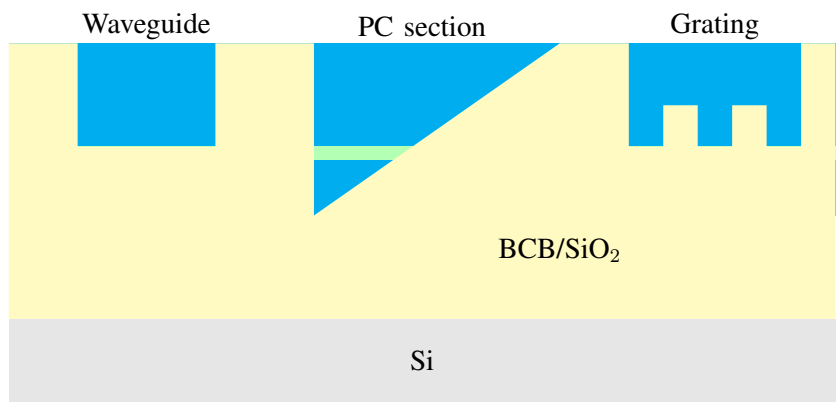

(c)

Fig. 4. Key steps in the fabrication process of a polarization converter on IMOS. (a) Initial layer stack for fabrication of polarization converters After vertical sidewall definition, coverage by silicon nitride and directionally etching of silicon nitride. (b) After consecutive wet etching with $\mathrm{HCl}: \mathrm{H}_{3} \mathrm{PO}_{4}$ (1:4), $\mathrm{H}_{2} \mathrm{SO}_{4}: \mathrm{H}_{2} \mathrm{O}_{2}: \mathrm{H}_{2} \mathrm{O}(1: 1: 10)$ and again $\mathrm{HCl}: \mathrm{H}_{3} \mathrm{PO}_{4}(1: 4)$ and removal of the silicon nitride mask. (c) After bonding and removal of substrate and etch-stop-layer.

in exploring the performance of the converter around full conversion, we can use $L_{1}=2 L_{\pi}-L_{2}$, where $L_{n}$ is the length of the $n^{\text {th }}$ section and $L_{\pi}$ is the half-beat length of the two eigenmodes of the triangular section, as shown in [3]. The half-beat length is given by $L_{\pi}=\frac{\pi}{\left(\beta_{1}-\beta_{2}\right)}$, where $\beta_{1}$ and $\beta_{2}$ are the propagation constants of the two eigenmodes. For this device, the half-beat length is calculated to be 2.1 microns, and hence the length of the full device is 4.2 microns. If one considers the Poincaré sphere, it becomes evident that the lengths of the two sections to achieve full conversion are of unequal length with tilt angles unequal to exactly $22.5^{\circ}$, by considering:

$$
L_{1}=\frac{L_{\pi}}{\pi} \cdot\left(\arcsin \left(\frac{1}{(\tan 2 \theta)^{2}}\right)+\frac{\pi}{2}\right)=2 L_{\pi}-L_{2} .
$$

To determine the optimal lengths of the two sections $L_{1}$ and $L_{2}$ and improve the accuracy, further 3D-FDTD simulations
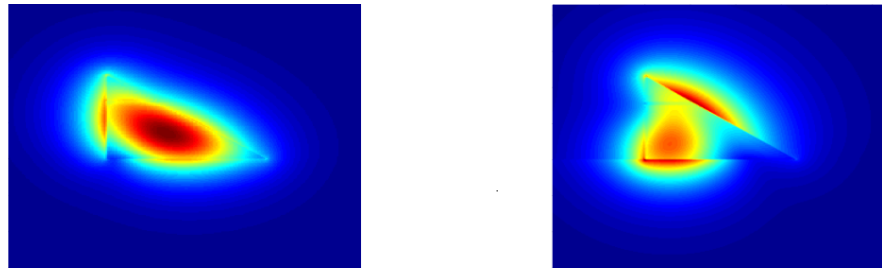

Fig. 5. Simulated field profile of the two eigen modes in the triangular section.

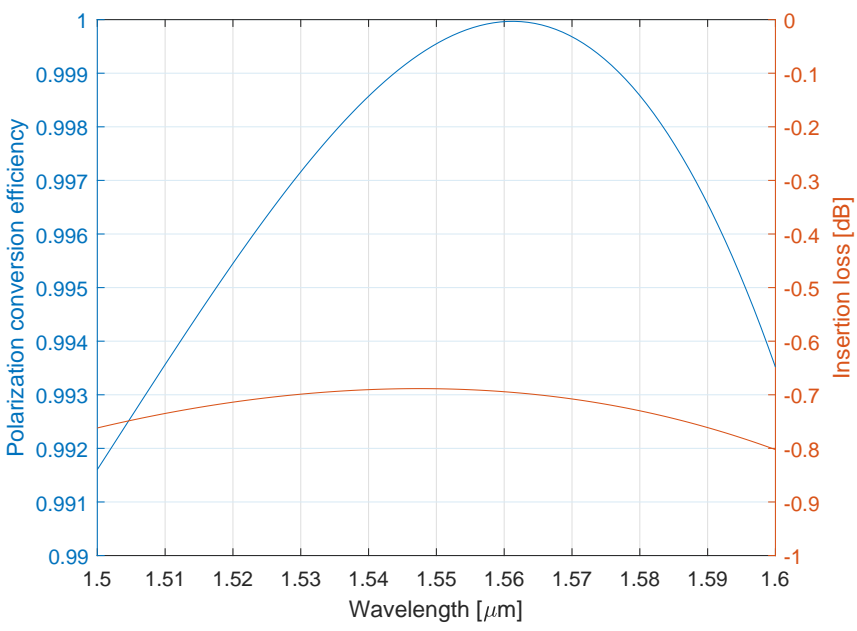

Fig. 6. Simulated efficiency and insertion loss for the optimized polarization converter.

are done, showing the optimal lengths for both sections to be $L_{1}=1.7$ microns and $L_{2}=2.3$ microns. We note that the length is slightly shorter than calculated from the mode simulation results. Two reasons which can explain this are the noted inaccuracy in the mode simulation, which was indicated by the TE fractions of the modes not adding up to exactly one, which one would expect. Secondly, a section of $200 \mathrm{~nm}$ long rectangular waveguide is introduced between the two rectangular sections, for fabrication reasons. The simulated conversion efficiency and insertion loss are shown in Fig. 6.

\section{Characterization}

In this section, the methodology for characterization of polarization conversion devices is first introduced. In the second part the results are shown for a polarization converter on the IMOS platform, and the new method is compared to a traditional measurement technique. The polarization conversion efficiency is defined as the fraction of light that has been converted to the orthogonal polarization by the converter. Alternately, one can consider the residual part that is not converted, the extinction ratio.

\section{A. Methodology}

1) Traditional method: Determining the conversion efficiency of a polarization converter on a platform such as the IMOS platform is often done by measuring both the TE and $\mathrm{TM}$ output of a converter, and using $\eta=\frac{P_{\mathrm{TM}}}{P_{\mathrm{TE}}+P_{\mathrm{TM}}}$ (if the input 


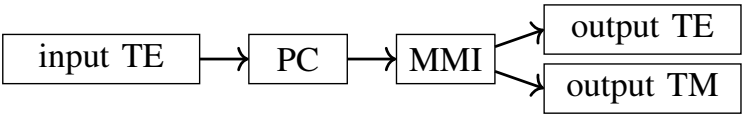

Fig. 7. Schematic view of a traditional characterization circuit for polarization converters.

was TE). A straightforward way to do this, is to have a TE input grating coupler, a polarization converter, a $1 \times 2$ multimode interference coupler for splitting the light 50/50, and a TE and TM grating coupler for the outputs, as shown schematically in Fig. 7. This structure offers the possibility for measuring the TE and TM output in two separate measurements. One has to compensate these measurements for the insertion loss of the MMI and the output grating couplers. The insertion loss of the input grating coupler is automatically compensated when calculating the conversion efficiency. To compensate for the insertion losses, calibration structures are required, which typically give an uncertainty of around $0.5 \mathrm{~dB}$ per grating coupler. This has a significant influence on the accuracy of the determined conversion efficiency, since this already adds up to around $20 \%$ uncertainty. Furthermore, the MMI behaves differently for the TE and TM mode due to a different effective refractive index for these modes. Due to symmetry, a $1 \times 2$ MMI is inherently balanced when used as a splitter, but it can result in a different insertion loss for both polarizations. The latter can be solved by either designing a polarization insensitive MMI, or by using two separate structures without the MMI, one with a TE output coupler and one with a TM output coupler. However, this can create an uncertainty in the measurement by itself, since one is actually measuring two structures in that case, introducing more uncertainties.

2) 4-port method: The method we introduce to accurately characterize the performance of our polarization converters is a measurement with four ports: two input ports and two output ports. The schematic view of the measurement circuit is shown in Fig. 8, and the design that is fabricated is shown in Fig. 9. For further reference, the input and output ports have been numbered, as shown in Fig. 8. We consider $P_{i j}$ the measured power at output $j$ when input $i$ is used, which, for all combinations of inputs and outputs results in

$$
\begin{aligned}
& P_{13}=\alpha_{1} \alpha_{3} \alpha_{\text {MMI }}^{2}(1-\eta) P_{\text {in }}, \\
& P_{14}=\alpha_{1} \alpha_{4} \alpha_{\text {MMI }}^{2} \eta P_{\text {in }}, \\
& P_{23}=\alpha_{2} \alpha_{3} \alpha_{\text {MMI }}^{2} \eta P_{\text {in }}, \\
& P_{24}=\alpha_{2} \alpha_{4} \alpha_{\text {MMI }}^{2}(1-\eta) P_{\text {in }},
\end{aligned}
$$

where $\alpha_{k}$ is the loss in input arm $k, \alpha_{\mathrm{MMI}}$ is the insertion loss of the MMI, $\eta$ is the polarization conversion efficiency and $P_{\text {in }}$ is the input power. We then introduce the parameter $C$ as

$$
C=\frac{P_{13} P_{24}}{P_{14} P_{23}}=\frac{(1-\eta)^{2}}{\eta^{2}} .
$$

The aforementioned losses are polarization dependent, but are compensated in Eq. 6 since all terms appear in both the numerator and denominator. From Eq. 6 it can be seen the parameter $C$ is independent of the insertion losses, propagation losses, and polarization sensitivity of in-circuit components,

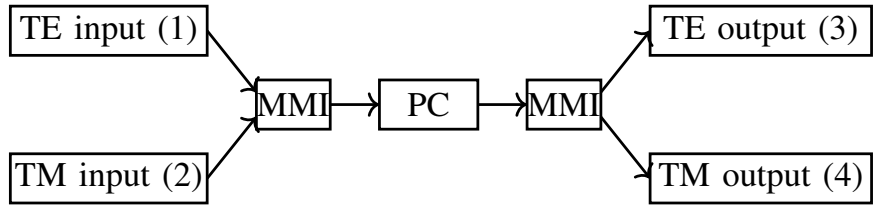

Fig. 8. Schematic of 4-port characterization circuit for the characterization of polarization converters.

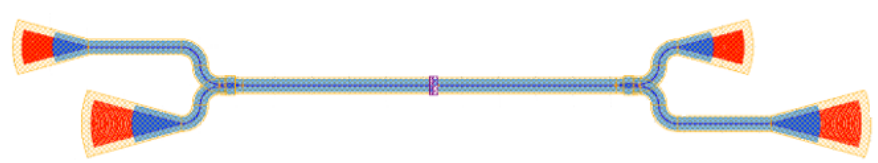

Fig. 9. Structure for a 4-port measurement. Input TE and TM grating couplers on the left, output TE and TM grating couplers on the right, polarization converter in the center, en two MMIs to connect the grating couplers to the polarization converter.

e.g. the MMIs, and only depends on the polarization conversion efficiency $\eta$. Identical MMIs are considered, however, non-identical MMIs will yield the same result, since the insertion losses are canceled out in Eq. 6. Rewriting the equation gives

$$
\eta=\frac{1}{1+\sqrt{C}}=\frac{1}{1+\sqrt{\frac{P_{13} P_{24}}{P_{14} P_{23}}}},
$$

which provides the polarization conversion efficiency independent of the losses in the grating couplers, the waveguides and the MMIs. An uncertainty in coupling from fiber to grating coupler is minimized by using an automated setup with high-accuracy alignment algorithms. The contribution of the uncertainty of the extinction ratio is therefore less than $0.2 \mathrm{~dB}$.

\section{B. Results}

1) Traditional method: The polarization conversion efficiency is calculated from measurement that are performed using the traditional method. Two structures with the same design for the polarization converter with both TE inputs and one TE and one TM output are measured. The results are first de-embedded by using calibration structures with TE and TM grating couplers to compensate for the polarization dependent insertion loss. For these results, $L_{1}=1.9$ microns, $L_{2}=2.65$ microns, and the length of the rectangular section $S$ is varied. In this way, the phase relation of the TE and TM polarized light at the input of $T_{2}$ will be different depending on the length of this section, and hence result in a different conversion efficiency. The calculated polarization conversion efficiency as a function of the length of section $S$ is shown in Fig. 10, at a wavelength of $1475 \mathrm{~nm}$, which was the peak of the transmission of the grating couplers for these structures. We notice the fit is quite poor, which can be explained by the aforementioned uncertainty in the compensation for the insertion losses of the grating couplers.

2) 4-port method: For this method, the length of the rectangular section, section $S$ in Fig. 2, between the two sections of the converter was kept constant at $200 \mathrm{~nm}$. Converters with length variations for $T_{2}$ have been measured over a 


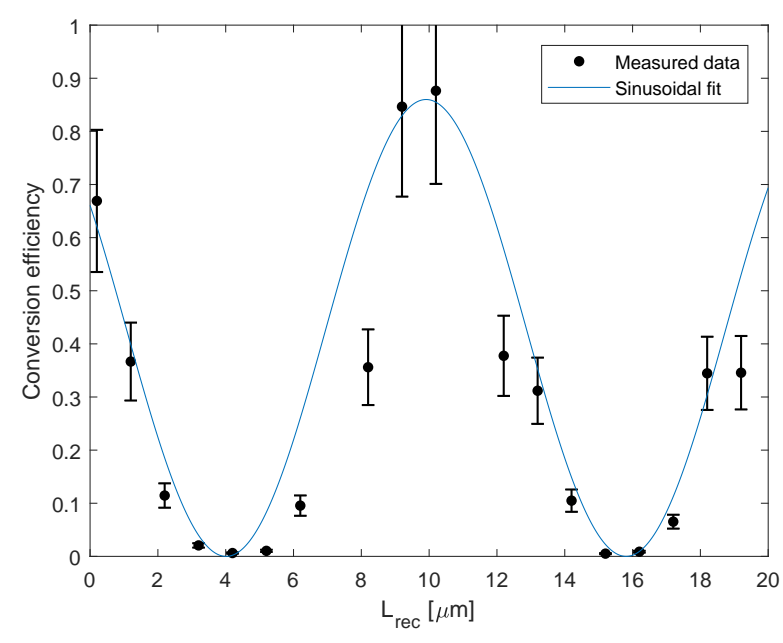

Fig. 10. Polarization conversion efficiency as a function of the length of the rectangular section at a wavelength of $1475 \mathrm{~nm}$. The scatter points represent measured devices and the blue line is a sinusoidal fit.

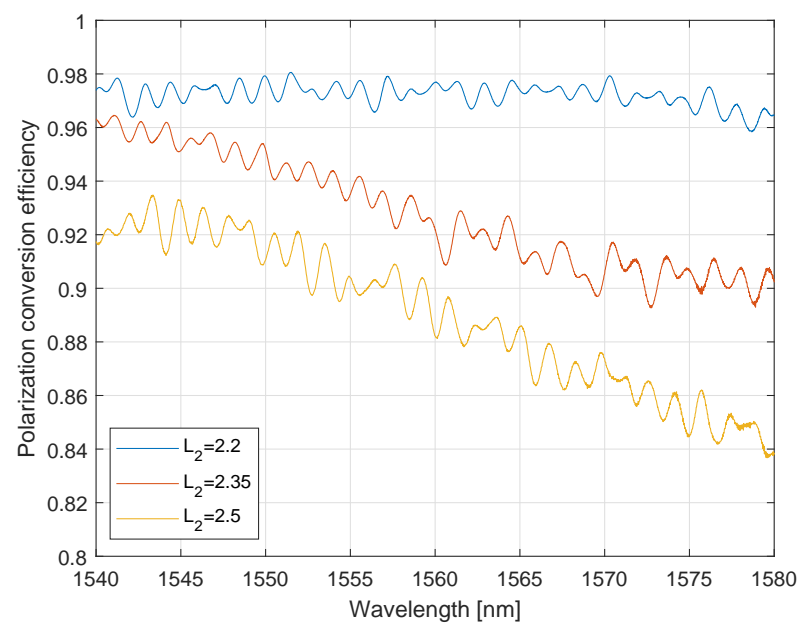

Fig. 11. Measured polarization conversion efficiency versus wavelength for three lengths $L_{2} . L_{1}$ is fixed at $1.7 \mu \mathrm{m}$.

wavelength range such that both TE and TM surface grating couplers have reasonable coupling efficiency, which is 1540 $1580 \mathrm{~nm}$ for this fabrication run.In Fig. 11, the measured polarization conversion efficiency (PCE) is shown. No direct comparison to simulated devices was performed, since there are several deviations from the optimal design, e.g. waveguide roughness, sidewall verticality and triangle height. The ripple on the PCE is caused by reflections in the circuit, which introduces $0.5 \%$ uncertainty in this method. Reducing these reflections can improve the accuracy of the method further. $97.5 \pm 0.5 \%$ PCE or $-16 \pm 0.9 \mathrm{~dB}$ extinction ratio is achieved, which is comparable to the state-of-the-art in silicon photonics [5], [6], [7]. The efficiency is above $96 \pm 0.5 \%$ (extinction ratio below $-14 \pm 0.9 \mathrm{~dB}$ ) for $1540-1580 \mathrm{~nm}$ for a total device length of $4.1 \mu \mathrm{m}$. In Fig. 12, the PCE at a wavelength of $1550 \mathrm{~nm}$ is shown for five devices with fixed $L_{1}$ and various $L_{2}$. A sinusoidal trend is expected, however, there is a

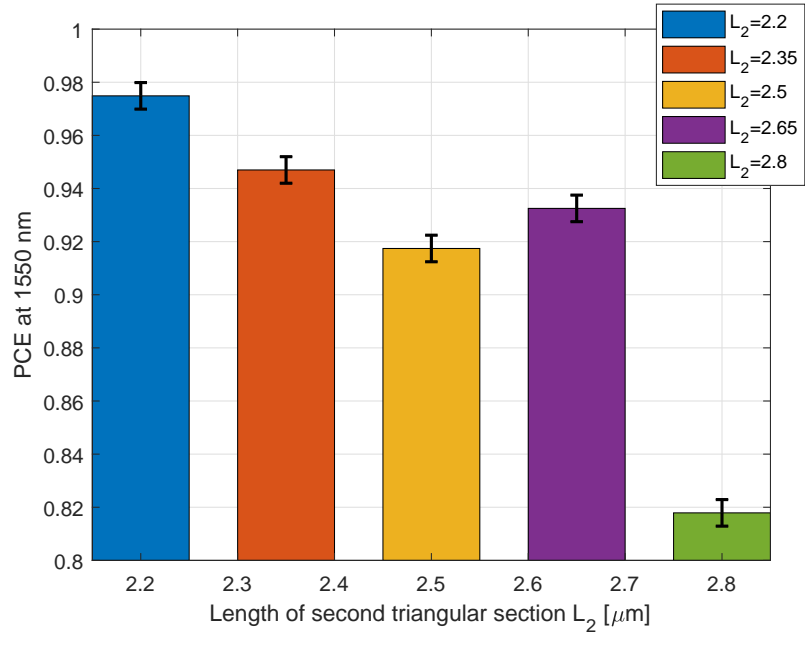

Fig. 12. Measured polarization conversion efficiency at $1550 \mathrm{~nm}$ for various lengths of the second triangular section. $L_{1}$ is fixed at $1.7 \mu \mathrm{m}$.

limited amount of structures available and there is an anomaly for $L_{2}=2.65 \mu \mathrm{m}$. After inspection of the structure, this is expected to be caused by residual InP after the wet etching of the triangular sections. Furthermore, it is likely the peak of the sinusoidal is not reached yet, which could mean a higher conversion efficiency can be achieved, as was predicted by the simulations. The reason for the peak being at a shorter device length is that the fabricated triangular sections are lower than designed. This is caused by the directional etch of the silicon nitride, shown in Fig. 4(a). A slight overetch is done to ensure that the horizontally covered sections are opened, etching away some of the silicon nitride on the vertical sidewall of the converter. The result is that the top $25 \mathrm{~nm}$ of the indium phosphide is etched away in the subsequent wet etching step, due to the absence of the hard mask in that part. This results in a drop in $L_{\pi}$ from around 2.1 microns to 1.85 microns, and hence a shorter device is required to achieve full polarization conversion.

\section{Discussion}

Although there is no direct comparison between the traditional characterization method and the 4-port method, we can see some differences in accuracy. For the traditional method, for which the results are shown in Fig. 10, it becomes clear that the accuracy is quite low, with some points deviating as much as $40 \%$ from the trend-line. If a sufficient amount of points are measured, the conversion efficiency can be obtained from the trend-line. However, if there is a deviation in one of the calibration structures, this will be a systematic error that will also appear in the trend-line. The 4-port method is shown to be independent of this type of errors, however one is still influenced by accurate alignment of the fiber to the grating coupler. However, with an automated setup, consecutive alignments are done with an accuracy much better than $0.1 \mathrm{~dB}$. The ripple on the conversion efficiency is around $0.5 \%$, which can be reduced by suppressing spurious reflections in the circuit, pushing the accuracy of the method even further. 


\section{CONCLUSION}

We present a new characterization method for polarization conversion devices and experimentally demonstrate the method on the IMOS platform. The accuracy of the traditional method is drastically improved to around $0.5 \%$ by introduction of this 4-port characterization method, by removing the uncertainties in insertion losses and polarization dependent losses in in-circuit components, leaving only the influence of spurious reflections in the circuit. The reported polarization conversion efficiency is as high as $97.5 \% \pm 0.5 \%$ corresponding to an extinction ratio of $-16 \pm 0.9 \mathrm{~dB}$ for a 4.1 microns long device.

\section{REFERENCES}

[1] S. Reniers, Y. Wang, K. Williams, J. Van Der Tol, and Y. Jiao, "Characterization of Waveguide Photonic Crystal Reflectors on Indium Phosphide Membranes," IEEE Journal of Quantum Electronics, vol. 55, no. 6, 2019.

[2] J. Van Der Tol, Y. Jiao, L. Shen, A. Millan-Mejia, V. Pogoretskii, J. Van Engelen, and M. Smit, "Indium Phosphide Integrated Photonics in Membranes," IEEE Journal of Selected Topics in Quantum Electronics, vol. 24, no. 1, pp. 1-9, 2018.

[3] J. Pello, "Building up a membrane photonics platform in indium phosphide," Ph.D. dissertation, Department of Electrical Engineering, Technische Universiteit Eindhoven, 2014.

[4] S. Adachi and H. Kawaguchi, "Chemical Etching Characteristics of (001)InP," Journal of the Electrochemical Society, vol. 128, no. 6, pp. 1342-1349, 1981.

[5] Y. Zhang, Y. He, X. Jiang, B. Liu, C. Qiu, Y. Su, and R. A. Soref, "Ultracompact and highly efficient silicon polarization splitter and rotator," $A P L$ Photonics, vol. 1, no. 9, 2016.

[6] D. Vermeulen, S. Selvaraja, P. Verheyen, W. Bogaerts, D. V. Thourhout, and G. Roelkens, "High Efficiency Broadband Polarization Rotator on Silicon-On-Insulator," Journal of the Optical Society of America, pp. 4244, 2010.

[7] A. Xie, L. Zhou, J. Chen, and X. Li, "Efficient silicon polarization rotator based on mode-hybridization in a double-stair waveguide," Optics Express, vol. 23, no. 4, p. 3960, 2015.

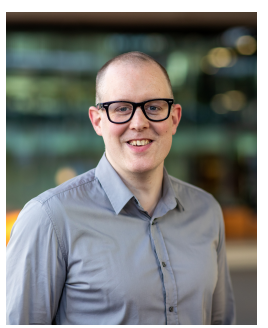

Sander Reniers was born in Hapert, The Netherlands. He obtained his B.Sc. and M.Sc. degree in electrical engineering from Eindhoven University of Technology in 2014 and 2017, respectively. He is currently working towards the Ph.D. degree at the same university. His research interests include waveguide reflectors and polarization diversity on the IMOS platform.

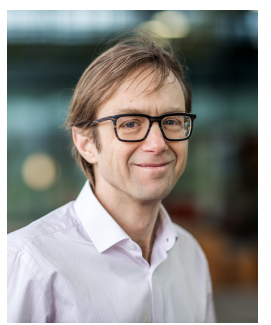

Kevin Williams received the B.Eng. degree from the University of Sheffield, Sheffield, U.K., and the Ph.D. degree from the University of Bath, Bath, U.K., in 1995. He moved to the University of Cambridge, Cambridge, U.K., in 2001 and was elected Fellow at Churchill College. His research interests are in the area of integrated photonic circuits. He was the recipient of a Royal Society University research fellowship at the University of Bristol, Bristol, U.K., in 1996. In 2006, he was the recipient of a European Commission Marie Curie Chair at the COBRA Institute, now known as the Institute for Photonic Integration at the Eindhoven University of Technology, The Netherlands. In 2011, he was the recipient of a Vici award from the Netherlands Organization for Scientific Research, where he has focused on photonic integration technology. He is the chair of the Photonic Integration research group at Eindhoven University of Technology.

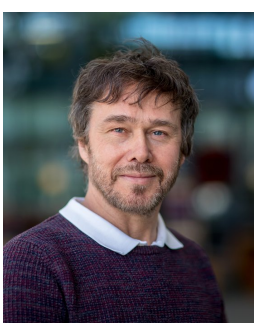

Jos van der Tol received the M.Sc and Ph.D degrees in physics from the State University of Leiden, The Netherlands in 1979 and 1985, respectively. In 1985 he joined KPN Research, where he became involved in research on integrated optical components for use in telecommunication networks. His research interest in this field have covered modelling of waveguides, design of electro-optical devices on lithium niobate and their fabrication. Furthermore he has been working on guided wave components on III-V semiconductor materials. He has also been active in the field of optical networks, focussing on survivability, introduction scenarios and management issues. Since July 1999 Dr. Van der Tol is working as an associate professor at the University of Technology Eindhoven in The Netherlands, where his research interests include opto-electronic integration, polarization issues, photonic membranes and photonic crystals. $\mathrm{He}$ is (co)author of more than 250 publications in the fields of integrated optics and optical networks, and has 25 patent applications to his name.

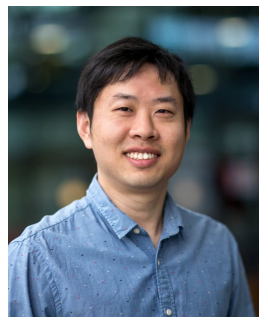

Yuqing Jiao was born in Hangzhou, China. He obtained $\mathrm{PhD}$ degrees from both Eindhoven University of Technology, the Netherlands, and Zhejiang University in China in 2013. Since then he continued his research at Eindhoven University of Technology. Since 2016 he is appointed as an assistant professor at the Institute of Photonic Integration (IPI, former COBRA Research Institute) of the Eindhoven University of Technology. His research topic is focused on a novel III-V based nanophotonic platform. He is focusing on ultrafast and strong light-matter interactions in sub-micron optical confinement. Applications span from optical interconnects, ultrafast photonic devices, to optical beam steering and optical sensing. He has strong background and expertise in a wide range of photonic materials (from silicon to III-V) and nanotechnologies. He has (co-)authored more than 30 international journal publications and 70 conference papers. $\mathrm{He}$ is a member of the IEEE Photonics Society and the Optical Society of America. Currently he serves as a board member of IEEE Photonics Society Benelux Chapter. 